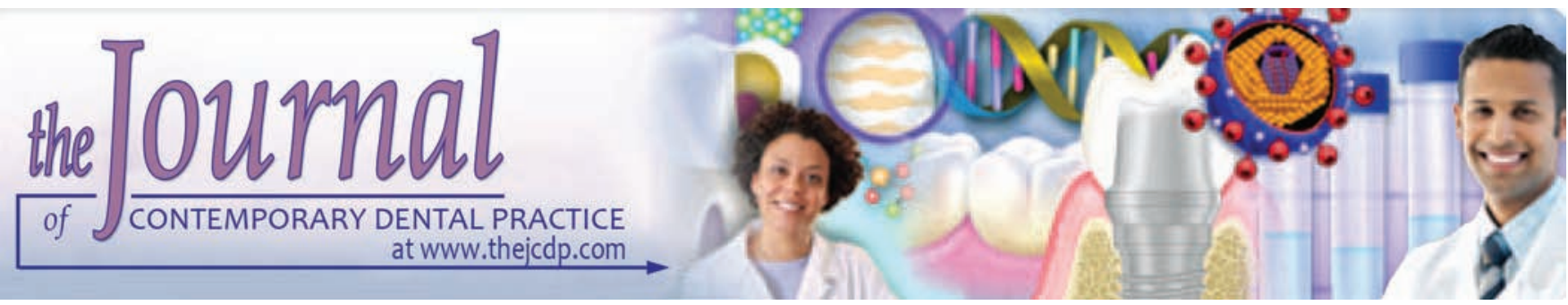

\title{
Endodontic Management of Dilacerated Maxillary Central Incisor fused to a Supernumerary Tooth using Cone Beam Computed Tomography: An Unusual Clinical Presentation
}

${ }^{1}$ Thilla S Vinothkumar, ${ }^{2}$ Deivanayagam Kandaswamy, ${ }^{3}$ Ganesh Arathi

${ }^{4}$ Sathishkumar Ramkumar, ${ }^{5}$ Gnanasekaran Felsypremila

\begin{abstract}
Aim: The purpose of this report is to present a case of endodontic management of a dilacerated maxillary central incisor fused to supernumerary tooth unusually appearing as a labial tubercle using cone beam computed tomography (СBCT) as a diagnostic aid.

Background: Anterior teeth are commonly encountered with aberrant anatomical variations in the crown, number of roots, and root canals. Fusion is an infrequent developmental abnormality in shape of the tooth caused by the union of two adjacent tooth germs. A complete knowledge of such complex anatomies is mandatory for a successful root canal treatment. It highlights the endodontic and restorative approach in the functional and esthetic rehabilitation of the involved tooth.
\end{abstract}

Case report: Three-dimensional imaging using CBCT was used in this case to differentiate single labial canal and palatal C-shaped canal. The endodontic treatment was performed with the aid of dental operating microscope, and the root canals were obturated with a combination of single cone, carrier based, and cold flowable gutta-percha obturation system. The tooth remained asymptomatic without reinfection and symptoms of failure for 18 months.

Conclusion: Cone beam computed tomography is indispensable in treatment planning for cases where the radiographs cannot reveal useful information regarding the root canal shape. Postobturation $\mathrm{CBCT}$ has helped in avoiding further retreatment of the tooth, which otherwise is a challenging task.

${ }^{1}$ Department of Restorative Dental Sciences, College of Dentistry, Jazan University, Jazan, Kingdom of Saudi Arabia

2,3,5 Department of Conservative Dentistry and Endodontics Faculty of Dental Sciences, Sri Ramachandra University Chennai, Tamil Nadu, India

${ }^{4}$ Private Dental Clinic, Chennai, Tamil Nadu, India

Corresponding Author: Thilla S Vinothkumar, Department of Restorative Dental Sciences, College of Dentistry, Jazan University, Jazan, Kingdom of Saudi Arabia, e-mail: vinothkumar_ ts@yahoo.com
Clinical significance: The use of sophisticated equipment, such as dental operating microscope and СBCT has helped in accurate diagnosis and treatment planning of fused central incisor respectively.

Keywords: Central incisor, Cone beam computed tomography, Dilacerations, Fusion, Root canal, Supernumerary.

How to cite this article: Vinothkumar TS, Kandaswamy D, Arathi G, Ramkumar S, Felsypremila G. Endodontic Management of Dilacerated Maxillary Central Incisor fused to a Supernumerary Tooth using Cone Beam Computed Tomography: An Unusual Clinical Presentation. J Contemp Dent Pract 2017;18(6):522-526.

Source of support: Nil

Conflict of interest: None

\section{INTRODUCTION}

The maxillary central incisor often presents the endodontic practitioner with a series of anomalous anatomy, thereby posing difficulty in diagnosis and treatment planning. ${ }^{1,2}$ Challenging cases - such as lingual groove defects, fusion, gemination, twinning, and dens invaginatus - are known to influence the progress of treatment. Fusion, gemination, and twinning can be categorized as double teeth because of the involvement of two tooth germs. ${ }^{3}$ Endodontic treatment of such teeth involves thorough debridement of the entire pulp space followed by threedimensional obturation of the same with an inert filling material. ${ }^{4}$ This goal can be accomplished by having a thorough knowledge of the internal and external anatomic features of the root and their variations. ${ }^{5,6}$

Fusion also termed as synodontia or false gemination is defined as a complete or partial union between the dentin of two or more teeth depending on the stage of tooth development. ${ }^{7}$ In contrast, gemination is an incomplete attempt of one tooth germ to divide into two, ${ }^{8}$ whereas twinning is similar to gemination if the 
division is complete. The union of a supernumerary tooth and a normal tooth is called diphyodontic gemination.? Dens invaginatus is characterized by invagination of the enamel organ into the dental papilla before calcification of the dental tissues. ${ }^{8}$

These developmental anomalies in shape and number have overlapping features but different etiology, thereby posing difficulty in differential diagnosis and management. ${ }^{9}$ Dental fusion is relatively common in maxillary anterior region with predilection to primary dentition (permanent $-0.1 \%$; primary $-0.5 \%$ ). ${ }^{10-12}$ Fused teeth are normally prone to caries and periodontal problems due to the presence of scratches and gaps at the place of union. ${ }^{13}$ Recently, limited field of view (FOV) cone beam computed tomography (CBCT) has been recommended following intraoral radiograph as a preoperative imaging modality of choice for the initial treatment of teeth with high probability of extra canal complex morphology. ${ }^{14}$ Despite the complexity of malformation in double tooth, nonsurgical orthograde root canal therapy is considered to be adequate treatment option. ${ }^{3}$

This case report presents the endodontic management of a dilacerated maxillary central incisor fused to a supernumerary tooth unusually presenting as a labial tubercle with the use of CBCT and dental operating microscope.

\section{CASE REPORT}

A 20-year-old female patient was referred to the Department of Conservative Dentistry and Endodontics for the endodontic management of an asymptomatic maxillary right central incisor from the Department of Orthodontics. On clinical evaluation, the anterior teeth were proclined with uniformly distributed spacing, and the number of teeth in the arch was normal (Fig. 1A). The dimensions of \#11 were normal and symmetrical except for a labial tubercle demarcated from the remaining surface with a discontinuity in enamel in the form of pit located in the middle-third of the labial surface (Fig. 1A). There was no mobility, swelling, or sinus tract and the periodontal probing depths were within normal limits. All maxillary incisors were responding normally to pulp sensitivity test except \#11, which was not responding to both electrical and cold pulp testing. Nevertheless, the patient could not give a definite history of trauma. A periapical radiograph revealed an unclear root canal morphology with the in-folding of enamel and dentin extending into the root with diffuse periapical radiolucency measuring approximately $4 \times 3 \mathrm{~mm}$ (Fig. 1B). Based on the clinical and radiographic features, a provisional diagnosis of labial dens invaginatus in maxillary right central incisor (\#11) with pulp necrosis and asymptomatic chronic apical periodontitis was made. The differential diagnosis for the bizarre root canal morphology was fusion with supernumerary tooth, gemination, and twinning.

A limited FOV CBCT was performed using Planmeca ProMax 3D (Planmeca USA Inc., Roselle, Illinois, USA) with the following technical parameters: FOV $68 \times 42 \mathrm{~mm}$, effective exposure time of 10.8 seconds (pulsated), tube voltage 54 to $90 \mathrm{kV}, 1$ to $14 \mathrm{~mA}$ (pulsated mode) with an estimated radiation dosage of 30 to $80 \mu \mathrm{Sv}$ (microSievert) and slice thickness of $0.2 \mathrm{~mm}$.

The CBCT images revealed a short supernumerary tooth fused onto the labial surface of maxillary central incisor with two separate root canals. The axial views of the CBCT scan showed a single round labial root canal pertaining to the supernumerary tooth and a palatal canal, which was elongated mesiodistally mimicking C-shaped canal in the crown and coronal third of the root due to compression (Fig. 1C). However, the remaining portion of the palatal root canal was round till the apex (Fig. 1D). Sagittal views revealed the labially fused supernumerary tooth against \#11 with individual canals giving a typical appearance in the coronal portion (Fig. 1E). In addition, the palatal root was dilacerated in the labiopalatal aspect due to pressure exerted from the overlying supernumerary tooth at the time of development. In coronal section, the root canal morphology was inconclusive; however, the extent of radiolucency with well-circumscribed radiopaque borders was clearly appreciable (Fig. 1F). Based on the clinical and CBCT findings, the confirmatory diagnosis was made as fusion of maxillary right central incisor (\#11) and supernumerary tooth with pulp necrosis and asymptomatic chronic apical periodontitis. The tooth was planned for nonsurgical endodontic treatment after obtaining the patient's consent.

An endodontic access was performed with No. 2 round bur and nonend-cutting tapered diamond abrasive (EX-24; Mani Inc., Tochigi, Japan) under rubber dam isolation without local anesthesia (Fig. 1G). The labial approach was preferred than the conventional palatal approach to obtain straight line access of both the canals. The labial and palatal canals were negotiated and their patency was confirmed using an endodontic explorer and a size $15 \mathrm{~K}$ file along with dental operating microscope (Revelation; Seiler Instruments, St. Louis, MO, USA) (Fig. 1H). The working length (labial - $21 \mathrm{~mm}$; palatal - $27 \mathrm{~mm}$ ) was established with electronic apex locator (Root ZX; J Morita Co., Osaka, Japan). The axial views of the CBCT scans were carefully observed to measure the residual dentin thickness around both the root canals. The apical size and taper of the root canal preparation were decided based on the criteria that the residual dentin thickness should exceed the one-third of the root dimension. ${ }^{15}$ The labial canal was prepared by crown-down approach using Race 0.06 nickel-titanium 
rotary endodontic instrument (FKG Dentaire, La Chauxde-Fonds, Switzerland) up to size 30 with digital torque control motor (X-Smart, Dentsply Maillefer Instruments SA, Ballaigues, Switzerland). The C-shaped canal was instrumented with stainless steel $\mathrm{K}$ files till size 20, followed by Race 0.06 instruments up to size 40 according to the manufacturer's instruction. The isthmus region was carefully instrumented by anticurvature filing technique using K-file up to size 25 to avoid perforation. ${ }^{15}$ During instrumentation, the canals were irrigated with copious amount of $3.0 \%$ sodium hypochlorite, and ultrasonic activation was done to ensure complete debridement of the C-shaped segment of the canal. Intracanal medicament of calcium hydroxide and iodoform (Metapex; Meta Biomed Co. Ltd., Cheonju, Korea) was given for a week. The intracanal medicament was removed followed by final irrigation with $17 \%$ ethylenediaminetetraacetic acid and sodium hypochlorite. The canals were dried with paper points and obturation of the labial root canal was performed with 0.06 taper gutta-percha cones and resin sealer (AH Plus; Dentsply Maillefer Instruments SA, Ballaigues, Switzerland). The palatal C-shaped canal was obturated using a combination of carrier-based
(Thermafil; Dentsply Tulsa dental products, Tulsa OK) and cold flowable (Guttaflow; Coltene Whaledent Inc., Langenau, Germany) gutta-percha obturation system according to the manufacturer's instruction. Following the resin sealer application, the mesial extent of the palatal C-shaped canal was obturated using size 40 Thermafil obturator in order to ensure complete apical seal. The remaining portion of the root canal was backfilled threedimensionally with guttaflow and the access was sealed with CavitG (3M ESPE, Seefeld, Germany) following vertical condensation of gutta-percha as evident in the periapical radiograph (Fig. 1I). Postobturation limited FOV CBCT examination (Figs 1J and $\mathrm{K}$ ) was performed after 3 weeks to rule out overextended obturation as the patient was experiencing postoperative pain for 3 weeks. ${ }^{14}$ Once the tooth was asymptomatic, the root canal orifice was sealed with resin-modified glass ionomer cement (Fuji II LC; GC Corporation, Tokyo, Japan). The labial tubercle was esthetically recontoured and the access cavity was restored with resin composite (Fig. 1L) before orthodontic treatment. One month follow-up showed satisfactory observation on clinical and radiographic examination. Eighteen months follow-up of the case did not reveal any
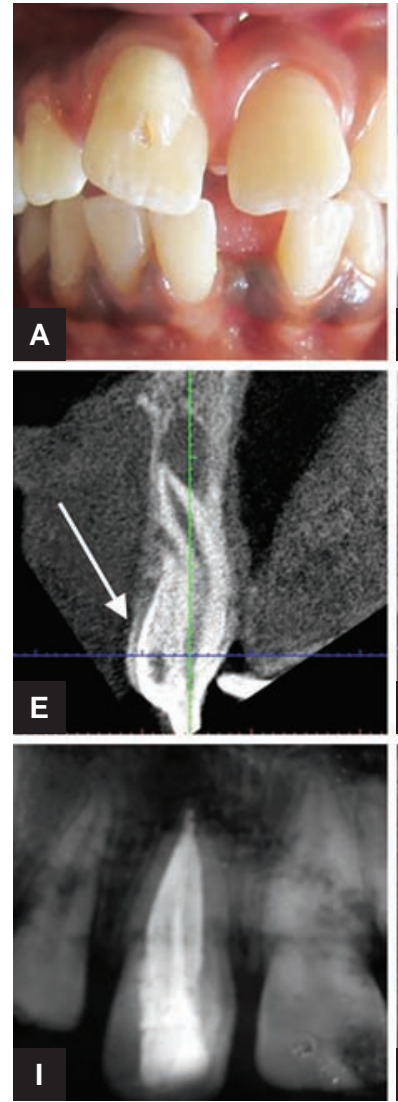
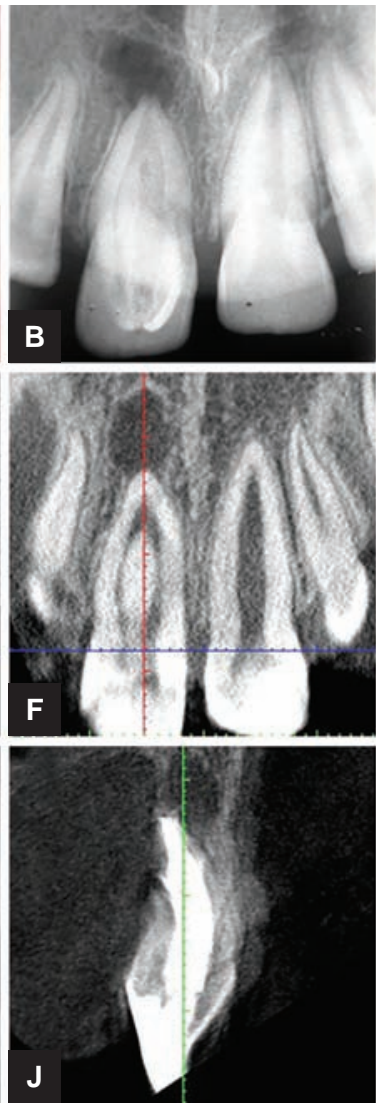
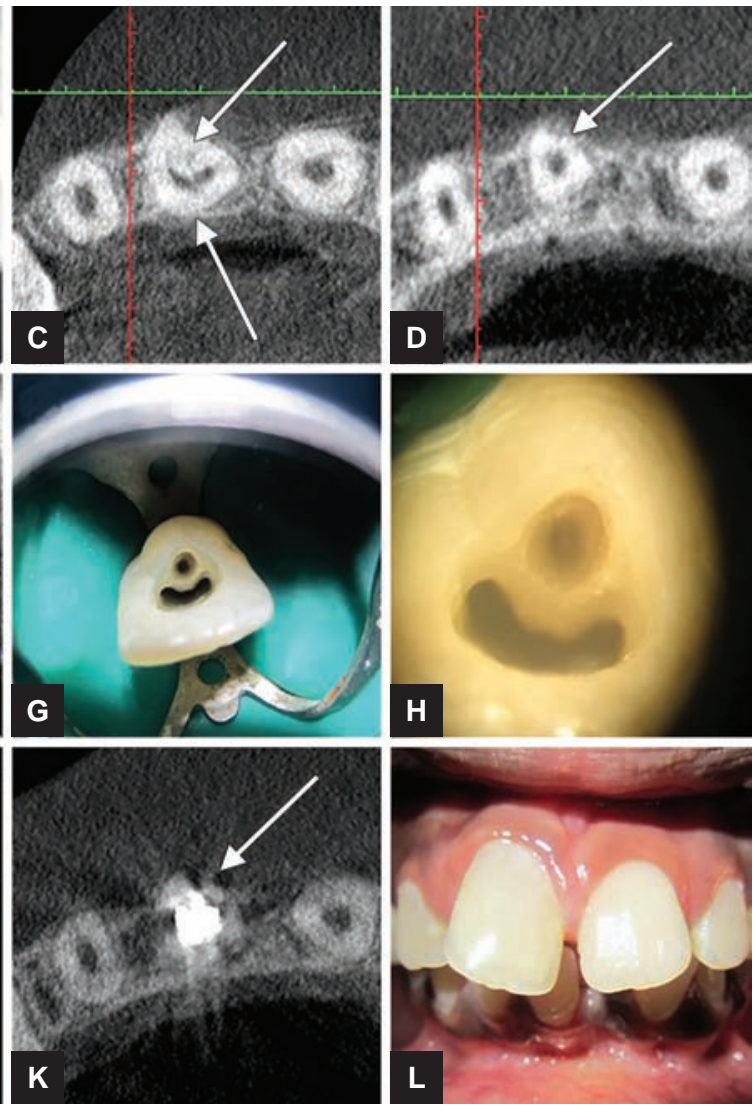

Figs 1A to L: Right maxillary central incisor labially fused to a supernumerary tooth: (A) Preoperative photograph showing the pit on labial surface; (B) preoperative periapical radiograph; (C) axial CBCT image of the cervical region showing two canals; (D) axial CBCT image of the apical third of the root showing single canal; (E) sagittal CBCT image of the crown; (F) coronal CBCT image; (G) labial access cavity showing palatal C-shaped canal; (H) microscopic view of the access cavity; (I) postobturation radiograph; (J) postobturation sagittal CBCT image; (K) postobturation coronal $\mathrm{CBCT}$ image; and $(\mathrm{L})$ postoperative photograph showing the final esthetic contouring with composite restoration 
signs of recurrent apical periodontitis and the tooth was completely asymptomatic.

\section{DISCUSSION}

The etiology of fusion is not exactly known. The physical forces that are formed during development may cause contact between the tooth germs, thereby resulting in necrosis of the epithelium between them, leading to fusion. ${ }^{11,16}$ Other possible causes are lack of space between the tooth germs during development, developmental aberrations during morphodifferentiation of the tooth bud, local metabolic disturbances, viral infection during pregnancy, thalidomide intake during pregnancy, and genetic inheritance. $^{12,17}$

Despite the fact that fusion and gemination are two different entities, it is often difficult to differentiate clinically, especially in cases of fusion between a permanent tooth and supernumerary tooth. ${ }^{3}$ Clinically, gemination most often presents two crowns, whereas the fused teeth possess large crown with radiographic one or two pulp chambers and root canals when viewed in the radiograph. ${ }^{11}$ When the anomalous fused tooth is considered as one unit, the number of teeth in the arch will be less by one count. In contrast, the dimensions of \#11 in our case were symmetric with that of \#21 except for a labial tubercle and tooth count was normal. Therefore, case history, clinical and radiographic examination are indispensable for accurate diagnosis of the condition. ${ }^{11}$

The fused teeth do not require any treatment as they are usually asymptomatic, unless they exhibit interference with occlusion, unacceptable esthetic appearance, and pulpal involvement. ${ }^{9}$ Depending on the location and the associated pathology, a comprehensive treatment plan including both the endodontic and periodontal procedures has (can be) been recommended. ${ }^{12}$ Surgical separation of the supernumerary tooth, which is fused to the permanent teeth, surgical extraction of unwanted part followed by orthodontic alignment is one of the suggested treatment options. Bicuspidization is an effective alternate treatment option in case of posterior teeth. However, it has given rise to various complications, such as external resorption and replacement resorption, ankylosis, loss of periodontal attachment, deep periodontal pocketing with persistent inflammation, and devitalization of the retained tooth section. ${ }^{18}$ In the present case, the labial pit with discontinuity in enamel and exposed dentinal tubules could be a source of bacterial penetration into the pulp, thereby resulting in pulpal necrosis and periapical lesion of endodontic origin. In the present case report, proclination with labial tubercle was a primary contributing factor toward unacceptable esthetics and interference with the placement of brackets during the orthodontic treatment. Hence, nonsurgical endodontic treatment was performed to manage the condition in a noninvasive manner, thereby avoiding the above-mentioned complications.

Cone beam computed tomography is a very useful tool in three-dimensional analysis of complex root canal anatomy, such as C-shaped root canals. ${ }^{19}$ Generally, the failure of Hertwig's epithelial root sheath to fuse during the development of tooth bud is the probable reason for the formation of C-shaped root canals in any teeth. ${ }^{15}$ However, the pressure exerted by the supernumerary tooth bud during the development of central incisor has resulted in formation of C-shaped root canal in our case. The conventional radiographs with two-dimensional images provided limited information that was inadequate for making a decision in the treatment plan. Hence, a preoperative limited FOV CBCT examination of the anterior maxilla was made to ascertain the exact three-dimensional complex anatomy and root canal morphology. ${ }^{14}$ The typical appearance of the fused maxillary central incisor and supernumerary tooth when viewed in the sagittal view may be considered for diagnosing other cases in future. Despite the advantages of three-dimensional imaging in assessing complex root morphology, their use is restricted to cases in which intraoral periapical radiographs are not informative. ${ }^{10}$ The combination of dilaceration and C-shaped root canal anatomy of the central incisor has increased the complexity of the case in diagnosis and treatment planning. The use of dental operating microscope has resulted in precise and sophisticated delivery of treatment methods without compromising the tooth structures and causing any endodontic mishaps. ${ }^{20}$

Generally, the root dimensions of maxillary central incisor are relatively more. However, the apical size and taper of the root canal preparation were critical due to the presence of supernumerary tooth, palatal C-shaped canal, and dilaceration. ${ }^{15}$ Despite the complete fusion of dentin except for the apical third of the supernumerary tooth as observed in the CBCT images, the instrumentation in the isthmus region was restricted to size 25 to avoid perforation or communication with the periodontium. The isthmus area can be effectively cleaned using ultrasonics or sonics combined with mechanical instrumentation. ${ }^{21}$ However, there was no necessity for ultrasonics in our case as the palatal C-shaped canal was continuous mesiodistally with a narrow isthmus.

Postobturation CBCT was taken and analyzed to rule out the pain due to overfilling of the root canals and to assess the quality of three-dimensional obturation. ${ }^{14}$ Carrier-based obturators possess the piston-like effect during placement which increases the likelihood of overfilling. ${ }^{22}$ Retreatment of root canals with such complex anatomy would have been otherwise a challenging task. However, CBCT scan should not be prescribed for the routine postobturation assessment of tooth. 


\section{CONCLUSION}

The advent of CBCT with a limited FOV and low effective dose equivalent to that of conventional radiography had made dental diagnosis simple and safe for the patient. This case report highlights the importance of anatomical variations that a central incisor could exhibit when fused with the adjacent tooth. A complete knowledge of diversities in root canal anatomy, appropriate use of diagnostic aids like CBCT, and regular use of dental operating microscope would facilitate successful endodontic treatment in such difficult cases.

\section{CLINICAL SIGNIFICANCE}

Although the incidence of fused teeth in permanent dentition is relatively less, the dentist must be alert when such cases are encountered to differentially diagnose and treat them with precise clinical acumen. Cone beam computed tomography is indispensable in treatment planning for cases where the radiographs cannot reveal useful information regarding the root canal shape.

\section{REFERENCES}

1. Rao Genovese F, Marsico EM. Maxillary central incisor with two roots: a case report. J Endod 2003 Mar;29(3):220-221.

2. Sponchiado EC Jr, Ismail HA, Braga MR, de Carvalho FK, Simões CA. Maxillary central incisor with two root canals: a case report. J Endod 2006 Oct;32(10):1002-1004.

3. Kremeier K, Pontius O, Klaiber B, Hülsmann M. Nonsurgical endodontic management of a double tooth: a case report. Int Endod J 2007 Nov;40(11):908-915.

4. Schwarze T, Baethge C, Stecher T, Geurtsen W. Identification of second canals in the mesiobuccal root of maxillary first and second molars using magnifying loupes or an operating microscope. Aust Endod J 2002 Aug;28(2):57-60.

5. Cantatore G, Berutti E, Castelluci A. Missed anatomy: frequency and clinical impact. Endod Topics 2006 Nov;15(1):3-31.

6. Vertucci FJ. Root canal morphology and its relationship to endodontic procedures. Endod Topics 2005;10(1):3-29.

7. Tannenbaum KA, Alling EE. Anomalous tooth development. Case reports of gemination and twinning. Oral Surg Oral Med Oral Pathol 1963 Jul;16:883-887.

8. Pindborg, J. Pathology of dental hard tissues. Copenhagen: Munksgaard; 1970. p. 30-64.

9. Tsesis I, Steinbock N, Rosenberg E, Kaufman AY. Endodontic treatment of developmental anomalies in posterior teeth: treatment of geminated / fused teeth - report of two cases. Int Endod J 2003 May;36(5):372-379.

10. Rani AK, Metgud S, Yakub SS, Pai U, Toshniwal NG, Bawaskar N. Endodontic and esthetic management of maxillary lateral incisor fused to a supernumerary tooth associated with a talon cusp by using spiral computed tomography as a diagnostic aid: a case report. J Endod 2010 Feb;36(2):345-349.

11. Faria MI, Borges ÁH, Carneiro SM, Silva-Filho JM, SemenoffSegundo A, da Cruz-Filho AM. Endodontic treatment of dental formation anomalies. Rev Odonto Cienc 2011;26(1):88-91.

12. Cho KM, Jang JH, Park SH. Clinical management of a fused upper premolar with supernumerary tooth: a case report. Restor Dent Endod 2014 Nov;39(4):319-323.

13. de Siqueira VC, Braga TL, Martins MA, Raitz R, Martins MD. Dental fusion and dens evaginatus in the permanent dentition: literature review and clinical case report with conservative treatment. J Dent Child (Chic) 2004 Jan-Apr;71(1):69-72.

14. AAE and AAOMR joint position statement: use of cone beam computed tomography in endodontics 2015 Update. J Endod 2015 Sep;41(9):1393-1396.

15. Bertrand T, Kim SG. Endodontic treatment of a C-shaped mandibular second premolar with four root canals and three apical foramina: a case report. Restor Dent Endod 2016 Feb;41(1):68-73.

16. Ferreira-Junior O, de Avila LD, Sampieri MB, Dias-Ribeiro E, Chen WL, Fan S. Lower third molar fused with a supernumerary tooth: diagnosis and treatment planning using conebeam computed tomography. Rev Gaucha Odontol 2014 Dec;62(4):453-458.

17. Kjaer I, Daugaard-Jensen J. Interrelation between fusions in the primary dentition and agenesis in the succedaneous permanent dentition seen from an embryological point of view. J Craniofac Genet Dev Biol 2000 Oct-Dec;20(4):193-197.

18. Olsen CB, Johnston T, Desai M, Peake GG. Management of fused supernumerary teeth in children using guided tissue regeneration: long-term follow up of 2 cases. Pediatr Dent 2002 Nov-Dec;24(6):566-571.

19. Felsypremila G, Vinothkumar TS, Kandaswamy D. Anatomic symmetry of root and root canal morphology of posterior teeth in Indian subpopulation using cone beam computed tomography: a retrospective study. Eur J Dent 2015 Oct-Dec;9(4):500-507.

20. AAE Special Committee to Develop a Microscope Position Paper. AAE position statement. Use of microscopes and other magnification techniques. J Endod 2012 Aug;38(8): 1153-1155.

21. Jafarzadeh $\mathrm{H}, \mathrm{Wu} \mathrm{YN}$. The C-shaped root canal configuration: a review. J Endod 2007 May;33(5):517-523.

22. Gluskin AH. Mishaps and serious complications in endodontic obturation. Endod Topics 2005 Nov;12(1):52-70. 